\title{
Barriers in Managing Acute Ureteric Colic Clinical Review and Commentary
}

\author{
Bodie Chislett ${ }^{\prime}$, Liang G Qu (iD ${ }^{1,2}$ \\ 'Department of Urology, Austin Health, Heidelberg, VIC, Australia; ${ }^{2}$ Young Urology Researchers Organisation (YURO), Melbourne, Australia \\ Correspondence: Bodie Chislett, Tel +61409171823, Email bodiechislett@gmail.com
}

\begin{abstract}
With the global prevalence of urolithiasis increasing, the presentation of acute ureteric colic to emergency departments (ED) poses a significant burden on healthcare systems globally. Management strategies for ureteric colic encompass medical expulsion therapy and various interventional modalities aimed at urinary diversion or definitive stone management. By examining potential or established barriers to managing acute ureteric colic, we can minimise strain on healthcare providers while maintaining patient outcomes. This review aims to assess barriers to the management of acute ureteric colic through a comprehensive overview of the current literature. Acute ureteric colic barriers will be assessed throughout a patient's disease progression, borrowing a conceptual framework used to assess barriers in cancer care management. Barriers will be discussed in the context of patient-centred access to healthcare, clinical evaluation and diagnosis, and management. Numerous barriers to healthcare have been identified throughout the natural course of acute ureteric colic, both specific and non-specific. Patient-centred barriers typically arise during the initial onset of acute ureteric colic. Originating from patient awareness and access to healthcare, they include barriers founded on race inequalities, cultural beliefs, geographic location, transportation, and the concept of a universal standard of healthcare. Having accessed healthcare, barriers in the management of acute ureteric colic next occur during the clinical evaluation and diagnosis period. These are typically associated with clinical assessment or diagnostic imaging delays, including underutilisation of ultrasound, nurse-led pathways for faster clinical reviews, and general ED delays. The final period during acute ureteric colic management correlates to clinical management. The inherent unpredictable course of ureteral stones leads to poor prognostication and failed initial management modalities. Additionally, this period deals with periprocedural delays and preventative health. Barriers to the management of acute ureteric colic arise during a patient's journey through accessing healthcare. Reviewing barriers allow further research into areas requiring modification to expedite care and improve outcomes.
\end{abstract}

Keywords: colic, barriers, management, improve, limitations, stones

\section{Introduction}

The global incidence of urolithiasis is between $1 \%$ and $20 \% .{ }^{1}$ Multiple variables contribute to this broad range, including age, sex, gender, ethnicity, and geographic location, where Western countries and regions with higher ambient temperatures have greater prevalence. ${ }^{1}$ Urolithiasis typically presents as acute ureteric colic, where the passage of stones formed within the renal pelvis causes a distal obstruction. Pain arises from the obstruction of urinary flow, where an accumulation of urine proximal to the obstruction causes increased pressure on vessel walls. ${ }^{2}$ Consequently, surges in prostaglandins cause arterial vasodilatation, increased ureteric oedema, and smooth muscle spasming.

Whilst often initially presenting within emergency departments (ED), the management of ureteric colic typically requires either subsequent admittance into a hospital or outpatient management. Initial management strategies historically employ temporising measures to manage pain and assist the spontaneous passage of ureteral stones, allowing for the abrogation of surgery where possible. Management strategies encompass medical expulsion therapy, and various interventional modalities aimed at urinary diversion or definitive stone management. Percutaneous nephrostomies and ureteral stents are forms of urinary diversion, with definitive ureteral stone management being either extracorporeal shockwave therapy or ureteroscopy and laser lithotripsy (URS). Expectant management is typically trialled initially to 


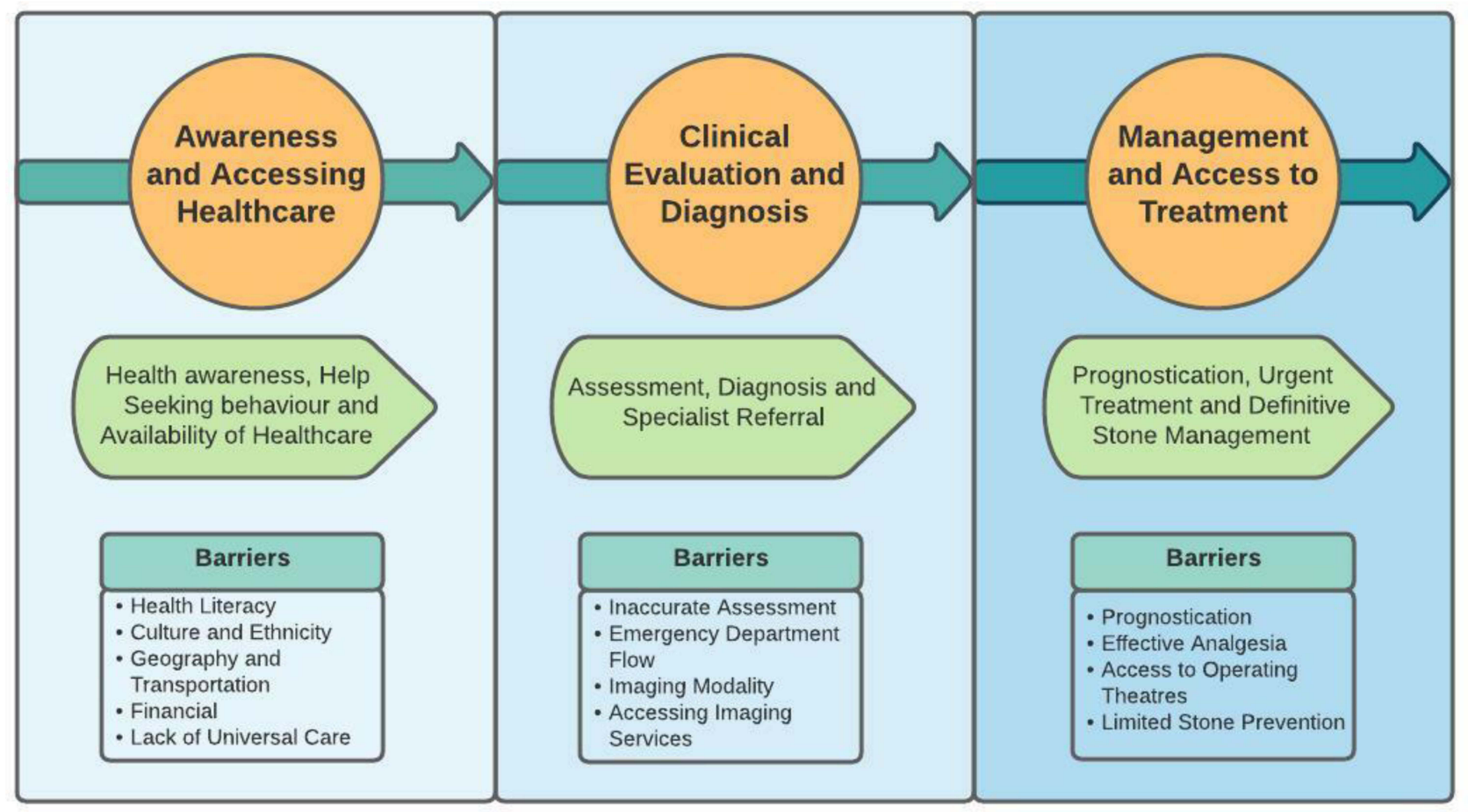

Figure I Chronological presentation of management barriers during acute ureteric colic.

Notes: Reproduced from Brand NR, Qu LG, Chao A, Ilbawi AM. Delays and barriers to cancer care in low- and middle-income countries: a systematic review. Oncologist.2019;24(I2):el37I-el 380, by permission of Oxford University Press. ${ }^{6}$

avoid more invasive intervention. Successful conservative management is dependent on multiple variables, though notably, the probability of spontaneous passage diminishes as the stone size increases. ${ }^{3,4}$ Refractory ureteric colic, in patients who are febrile or possess a solitary kidney, are absolute indications of ureteral stone intervention via diversion or definitive stone management.

With the global incidence and prevalence of urolithiasis increasing over consecutive decades, the importance of effective and efficient care is paramount for sustainability. ${ }^{1,5}$ By examining potential or established barriers to managing acute ureteric colic, we can minimise strain on healthcare providers while maintaining patient outcomes. Thus far, prior literature has not examined or outlined the various potential components that conceptually make up the barriers to ureteric colic management. Borrowing a conceptual framework frequently used to assess barriers in the management of cancer care, acute ureteric colic barriers will be assessed throughout a patient's progression (Figure 1). ${ }^{6}$ This review aims to assess barriers to the management of acute ureteric colic through a comprehensive overview of the current literature. Barriers will be discussed in the context of patient-centred access to healthcare, clinical evaluation and diagnosis, and management.

\section{Patient-Centred Barriers}

\section{Health Literacy, Race and Cultural Barriers}

Access to healthcare is impacted by several factors including availability of services, geographical access, and financial affordability. While these concepts have previously been examined in the broader context of other health conditions, there is a paucity of the literature directly addressing the management of acute ureteric colic. Despite the lack of relevant literature, obstacles may exist during an individual's ability to access management for acute ureteric colic. The importance of early access to care extends beyond just shortening the time interval to presentation. Reducing time to implementation of medical expulsion therapy may increase expulsion success and reduce subsequent requirements for procedural intervention. ${ }^{7}$ 
Health literacy and willingness to access healthcare play pivotal roles in managing all health conditions. Necessary skills and behaviours are required to make appropriate health decisions and successfully navigate healthcare paradigms. Poor health literacy leads to less access to healthcare and more frequent use of emergency healthcare. ${ }^{8}$ National data from the United States indicate approximately one-third of Americans have limited health literacy. In relation to urolithiasis, these low rates of health literacy should be improved to reduce the barriers to timely diagnosis and treatment. The impact of poor health literacy has previously been reported in a study from Nigeria, where a low level of awareness of urinary stone disease has been suggested to impact clinical management. ${ }^{9}$ Interventions should be explored to improve patient health literacy for the management of ureteric colic.

Cultural and religious beliefs may affect access to healthcare. Similarly, marginalised communities may have mistrust in mainstream healthcare systems. This sentiment may be particularly prevalent within indigenous communities, where experiences of discrimination and poor communication with healthcare professionals impact outcomes. Davy et al have highlighted a multitude of barriers to accessing healthcare by indigenous communities in Australia, United States, Canada, New Zealand and South America. ${ }^{10}$ While there is currently a lack of evidence to the degree that cultural and religious beliefs may have on managing ureteric colic specifically, it may be inferred that these patient-related barriers may still apply. Discrepancies between race and ethnicity may also exist in the timing of ureteral stone surgery, where Black and Hispanic patients wait up to seven days longer. ${ }^{11}$ These patients also presented more frequently to the ED with acute ureteric colic, in relation to likely delays in surgery. Broadly speaking, race and ethnicity may impact on many other surgical disciplines, with delays being described throughout the management path. ${ }^{12,13}$

\section{Geographical Access and Transportation Barriers}

Geographical accessibility principally impacts those residing within remote and regional settlements. Countries with a smaller population density and more significant landmass have difficulty providing a consistent standard of care, such as Canada and Australia. ${ }^{14}$ In an attempt to tackle such geographical issues, there has been a tendency for "regionalisation". Unfortunately, regionalisation may lead to additional travel times for many. Patients living further away are known to underutilise healthcare such as outpatient appointments and have more difficulty accessing tertiary hospital ED. ${ }^{15}$ Prior studies have already identified associations between travel distance and factors related to stone care, including negatively impacting quality of life outcomes. ${ }^{16,17}$ A review from 2013 identified six papers showing distance travelled as a perceived barrier to healthcare access. ${ }^{18}$ Similarly, the authors identified literature showing that health outcomes of urban populations are impacted by the availability of transport. These findings highlight how urban and rural settings both have transportation barriers, despite alternative transit options. Implementation of telehealth provides a practical solution, with utilisation increasing throughout the era of COVID-19. Novara et al identified six publications showing the successful application of telehealth for stone disease during the pandemic. ${ }^{19}$

\section{Universal Care and Financial Access Barriers}

The World Health Organization has identified access to care as key to improving global health outcomes, including managing ureteral stones. ${ }^{20}$ Universal standards of urological care represent a barrier to the global management of ureteric colic. Metzler et al examined the differences in care between low- and middle-income countries (LMIC) and high-income countries (HIC). They reported that urologists in LMIC were less likely to perform the American Urology Association standard of care. As an example, $16 \%$ of urologists would perform an open ureterotomy for an 8-mm proximal ureteral stone. ${ }^{21}$ Comparably, $61 \%$ of HIC urologists and $37 \%$ of LMIC urologists would manage an 8 -mm proximal ureteral stone endoscopically. Urologists also reported experiencing barriers in either a patient's ability to pay, or obtaining insufficient remuneration. ${ }^{21}$ In addition to suboptimal care, patients in LMIC are more likely to experience delays associated with shortages in surgeons, anaesthetists and theatre time. ${ }^{22}$

A relationship between HICs, socioeconomic class and private health status invariably exists. Discrepancies at the time of definitive stone management also exist, with a study conducted in the United States suggesting insured patients compared to uninsured patients received surgery seven days earlier. ${ }^{11}$ Healthcare payer types have long been associated with wait times, including for urological procedures. ${ }^{20}$ Payer type may similarly be expected to cause delays in managing acute ureteric colic within ED. Hall et al highlighted the discrepancies between care for patients with prostate cancer, 
where presentations to private hospitals (odds ratio: $2.40,95 \%$ confidence interval [CI]: $2.11-2.72$ ) and having private health insurance $(1.77,1.56-2.00)$ significantly increased the likelihood of undergoing a radical prostatectomy. ${ }^{14}$ Currently, there are no recent reports in the literature assessing the management of acute ureteric colic, though socioeconomic class and health insurance status may affect care.

\section{Clinical Evaluation and Diagnosis Barriers}

\section{Clinical Review Barriers}

Ureteric colic is classically associated with debilitating pain, microscopic haematuria, and renal angle tenderness. ${ }^{2}$ Primary management focuses on prompt and effective analgesia, with the severity of pain necessitating many to present to hospitals. Barriers may therefore exist at patient presentation to ED. Interruptions in care or limited resources in ED are well reported, from bed access blockage, increased waiting times and delays in imaging. ${ }^{23,24}$ These barriers may subsequently impact the management of ureteric colic.

Some hospital EDs have adopted alternative renal colic pathways. Nurse-led fast-track pathways have been established to reduce patient waiting times, providing earlier clinical review, faster diagnostic imaging, and better analgesia. ${ }^{25}$ Nurse-led fast-track pathways have been shown to reduce the time to radiologist-reported imaging by 50 minutes. ${ }^{25}$

The era of COVID has necessitated a reimagining of ED practices, providing a new framework for clinicians in the management of acute ureteric colic. Global incidence of ureteric colic presentations has decreased, however the rate of complicated ureteral stones has increased. ${ }^{26}$ In a recent review by Abdel Raheem et al, several studies outlined clinical pathways or tiered triaging systems that may aid in the management of acute ureteric colic. ${ }^{27}$ These alternative pathways provide early identification and commencement of hospital-specific management tips, including analgesia regimes and review criteria, some using nurse-led protocols. Such systems could break down barriers in management, though further research into the area is required for the efficacy and generalizability of these interventions.

\section{Diagnosis Barriers}

Access to imaging can cause delays in the management of ureteric colic. Obstructed stones in the presence of infection, severe kidney injury, or complete ureteric obstruction are considered urological emergencies, where expedient use of imaging such as computed tomography (CT) may avoid delays in management. ${ }^{28}$ Between 1996 and 2007, Westphalen et al showed an increased use of CT from $4.0 \%$ to $42.5 \%$ in the diagnosis of nephrolithiasis. ${ }^{29}$ Despite being increasingly utilized, the reliance on advanced diagnostic imaging may increase the length of stay within ED. Kanzaria et al reported a 114-minute difference between those undergoing advanced diagnostic imaging compared to those who did not. ${ }^{30}$

Although CT offers greater sensitivity and comparable specificity, ultrasonography (US) as an alternative modality may be valid in certain scenarios, as recommended by the European Association of Urology (EAU). ${ }^{28}$ US is recommended as a first-line modality in suspected ureteric colic, though it is not as readily used. ${ }^{28,31}$ Identifying uncomplicated suitable patients for US first pathways is important. The use of predictive algorithms offers a means of identifying such patients, though they predominantly remain unadopted as most are invalidated with limitations for adaption into practice. ${ }^{5,32}$ US offers a feasible and accessible alternative to $\mathrm{CT}$ in suspected acute ureteric colic presentations. Whilst most patients undergoing invasive intervention will receive CT imaging, earlier US, if accessible, can lead to earlier management via conservative modalities. Additionally, US-first pathways do not delay the time to intervention. ${ }^{33}$ Multiple studies suggest CT does not improve patient-centred outcomes compared to US-first diagnostic pathways, including diagnosis rates or hospital admissions. ${ }^{29,31-34}$ However, numerous limitations may exist in preventing widespread adoption into standard practice. ${ }^{35}$ While acknowledging the dependency of US on operator skills, properly trained US scanning may be performed by the bedside by emergency physicians, providing flexibility while reducing wait times. Its use is highly specific when identifying moderate-to-severe hydronephrosis. ${ }^{36}$

Patients undergoing US as their initial imaging modality do, however, have an increased likelihood of additional imaging. Smith-Bindman et al demonstrated that $40.7 \%$ of the patients undergoing initial point-of-care US and $27.0 \%$ of the patient's undergoing initial radiology-led US underwent subsequent $\mathrm{CT}^{33}$ However, US-first pathways have been shown not to lengthen the time to intervention. ${ }^{33}$ Regardless, the use of US may exclude clinically insignificant stones 
measuring less than $5 \mathrm{~mm}$, and hence its underutilisation may be a barrier in reaching diagnosis. ${ }^{33}$ To combat delays and barriers faced in diagnosing ureteral stones, hospitals may consider prioritizing the upskilling of emergency physicians in point-of-care US and validating predictive models.

Accurate diagnosis is in part the cause of increased CT use, offering a higher degree of accuracy. Admittedly, in some regards the increased use of $\mathrm{CT}$ is justified, as misdiagnosis poses a barrier to the care of acute ureteric colic by providing incidental findings. False-negative rates in urinalysis also contribute to the misdiagnosis barrier, estimated to be approximately $5 \%$ of cases. $^{37}$

\section{Management Modalities and Access \\ Barriers in Prognostication}

The optimal time and criteria for intervention in the acute ureteric colic remain unclear, with significant variation in admission and intervention patterns. ${ }^{5}$ Uncertainty surrounding appropriate management and attempts to avoid invasive intervention often leads patients and clinicians to elect for a trial of conservative management, reflected in many guidelines. ${ }^{5,28,38}$ Failed conservative care can partially be attributable to poor prognostication, as ureteric colic is inherently unpredictable Conservative management frequently fails, as up to $40 \%$ of patients may experience difficulty in expulsion. ${ }^{5}$ Inaccurate prognostication for determining cases suitable for conservative management may be a substantial barrier to prompt and efficient care.

Predictive models prognosticating an individual's need for urologic intervention are not well elucidated or defined. Several studies have attempted to incorporate clinical, laboratory and radiological findings to identify patients needing admission or intervention. Unfortunately, like other predictive models, none have been widely adopted due to publication limitations or failed validation studies. $3,4,32,38$

\section{Expectant Management Barriers}

Timely and efficacious analgesia is the first step in the acute ureteric colic treatment algorithms. Delays in analgesia administration and control of pain may present an obstacle in subsequent management. Standardised analgesia regimes may improve time for analgesia administration. Steinberg et al reported a 30-minute reduction in time to effective analgesia (72 \pm 63 vs $37 \pm 42$ minutes, $p=0.003) .{ }^{39}$ Previously discussed fast-track pathways offer a viable solution, with similar outpatient protocols suggested for pain management. ${ }^{25}$ However, this may have limited success given the need for close clinical observation when administering opiates.

Suboptimal choice of analgesic plan may impede management. First-line therapy for managing ureteric colic is nonsteroidal anti-inflammatories (NSAIDs), with less evidence for the first-line use of opiates. ${ }^{28}$ While alternative analgesic modalities exist, NSAIDs and opiates remain the mainstay of analgesia in EDs for acute ureteric colic. Despite the superiority of NSAIDs in controlling ureteric colic associated pain when compared to opiates, and a more favourable side effect profile, $43 \%$ of patients receive an opiate, $70 \%$ have opiates prescribed following admission, and $50 \%$ received opiates before surgery. ${ }^{40-42}$ Additionally, as refractory pain is commonly an indication for emergency decompression, suboptimal analgesic regimes may lead to higher rates of invasive intervention. ${ }^{5}$ The inappropriate use of opiates and underutilisation of NSAIDs may lead to poorly controlled pain and the need for escalation in management.

\section{Interventional Management Barriers}

As many as $75 \%$ of admitted patients undergo urological decompressive intervention. ${ }^{5,11}$ Barriers on the day of intervention include delays in arranging, attending or performing the intervention. Human errors and system deficiencies are prime factors in contributing to delays. ${ }^{43}$ Where some patient-dependent factors such as fasting status or medication use are unavoidable in select cases, human error and system failings are subject to improvement. A prior study reported that more than $50 \%$ of surgical cases experienced a perioperative delay. ${ }^{44}$ Due to its high prevalence, the impact of periprocedural delays on subsequent patient, hospital and health-economic-related outcomes remains unquantified.

The allotment of operative time is considered a rare resource. Hospitals may increase available theatre time by electing for ureteral stent insertion and deferring definitive ureteral stone management. Clinical decisions are influenced 
by the increased risk and technical difficulty of primary procedures to delay definitive management. The presentation to emergency and/or the intervention scheduling after-hours is a dynamic resource deficiency. Called the "weekend effect", it has been shown to prolong the time to intervention or surgery in managing ureteral stones. ${ }^{45}$

\section{Definitive Stone Management, Preventative Medicine and Outpatient Management}

With the increasing prevalence and burden of disease, there is a need to limit presentations of acute ureteric colic through early definitive management and ensured follow-up. In a recent retrospective study of acute ureteric colic, only $23 \%$ of patients received a referral to a urologist, and only $71 \%$ of those patients attended a urology appointment, equating to $16 \%$ of patients attending a follow-up appointment. ${ }^{46}$ Many of the aforementioned topics discussed likely present a barrier to outpatient follow-up. They include geography, access to transportation, race/ethnicity, private health insurance and financial status.

In the recent era of COVID-19, some hospitals are reporting fewer ED presentations of acute ureteric colic. ${ }^{47}$ It may be hypothesised that the community prevalence still remains stable, suggesting patients may be forgoing or delaying treatment to avoid potential exposure to COVID-19. Based upon these data, Steinberg et al raise the possible notion of over-treating ureteric colic prior to COVID-19. ${ }^{47}$ These suspicions should prompt further review and investigation into pathways preventing presentations, such as through outpatient or fast-track pathways. These potential changes in practice habits may overcome the barriers in accessing limited resources.

\section{Conclusion}

Acute ureteric colic has a high prevalence and burden of disease, straining multiple areas of limited resources. To improve patient flow and care, potential barriers to managing acute ureteric colic have been outlined. These barriers exist throughout a patient's journey in accessing healthcare, from: patient-centred barriers, clinical evaluation and diagnosis barriers, and management barriers. Further research should be conducted into interventions that may address these potential barriers to expedite care and improve patient outcomes.

\section{Disclosure}

The authors report no conflicts of interest in this work.

\section{References}

1. Ziemba JB, Matlaga BR. Epidemiology and economics of nephrolithiasis. Investig Clin Urol. 2017;58(5):299-306. doi:10.4111/icu.2017.58.5.299

2. Golzari SEJ, Soleimanpour H, Rahmani F, et al. Therapeutic approaches for renal colic in the emergency department: a review article. Anesth Pain Med. 2014;4(1). Available from: https://pubmed.ncbi.nlm.nih.gov/24701420/

3. Moon YJ, Kim HW, Kim JB, Kim HJ, Chang YS. Distribution of ureteral stones and factors affecting their location and expulsion in patients with renal colic. Korean J Urol. 2015;56(10):717-721. doi:10.4111/kju.2015.56.10.717

4. Coll DM, Varanelli MJ, Smith RC. Relationship of spontaneous passage of ureteral calculi to stone size and location as revealed by unenhanced helical CT. Am J Roentgenol. 2002;178(1):101-103. doi:10.2214/ajr.178.1.1780101

5. Daniels B, Schoenfeld E, Taylor A, Weisenthal K, Singh DMC. Predictors of hospital admission and urologic intervention in adult emergency department patients with CT-confirmed ureteral stones brock. Physiol Behav. 2016;176(1):100-106.

6. Brand NR, Qu LG, Chao A, Ilbawi AM. Delays and barriers to cancer care in low- and middle-income countries: a systematic review. Oncologist. 2019;24(12):e1371-e1380. doi:10.1634/theoncologist.2019-0057

7. Sfoungaristos S, Kavouras A, Kanatas P, Duvdevani M, Perimenis P. Early hospital admission and treatment onset may positively affect spontaneous passage of ureteral stones in patients with renal colic. Urology. 2014;84(1):16-21. doi:10.1016/j.urology.2014.01.005

8. Hersh LS. Health literacy in primary care practice. Am Fam Physician. 2015;92(2):118-124.

9. Odoemene C, Okere PCN, Ugonabo M. Ureterolithiasis: management in an environment with limited facilities. Niger J Clin Pract. 2017;20 (5):622-628. doi:10.4103/njcp.njcp_14_16

10. Davy C, Harfield S, McArthur A, Munn Z, Brown A. Access to primary health care services for indigenous peoples: a framework synthesis. Int J Equity Health. 2016;15(1):1-9. doi:10.1186/s12939-016-0450-5

11. Brubaker WD, Dallas KB, Elliott CS, et al. Payer type, race/ ethnicity, and the timing of surgical management of urinary stone disease. $J$ Endourol. 2019;33(2):152-158. doi:10.1089/end.2018.0614

12. Bustami RT, Shulkin DB, O’Donnell N, Whitman ED. Variations in time to receiving first surgical treatment for breast cancer as a function of racial/ethnic background: a cohort study. JRSM Open. 2014;5(7):204253331351586. doi:10.1177/2042533313515863

13. Bradley EH, Herrin J, Wang Y, et al. Racial and ethnic differences in time to acute reperfusion therapy for patients hospitalized with myocardial infarction. J Am Med Assoc. 2004;292(13):1563-1572. doi:10.1001/jama.292.13.1563 
14. Hall SE, Holman CD, Wisniewski ZS, Semmens J. Prostate cancer: socio-economic, geographical and private-health insurance effects on care and survival. BJU Int. 2005;95(1):51-58. doi:10.1111/j.1464-410X.2005.05248.x

15. Chan L, Hart LG, Goodman DC. Geographic access to health care for rural medicare beneficiaries. J Rural Health. 2006;22(2):140-146. doi:10.1111/j.1748-0361.2006.00022.x

16. Brooks NA, Paul CJ, Ghareeb GM, Tracy CR. Pushing stones uphill: why patients are lost to follow-up after uncomplicated ureteroscopy. $J$ Endourol. 2017;31(2):135-140. doi:10.1089/end.2016.0675

17. Hill C, States U, Hill C, et al. The effect of travel distance on health-related quality of life for patients with nephrolithiasis. Can Urol Assoc J. 2019;14(4):99.

18. Syed ST, Gerber BS, Sharp LK. Traveling towards disease: transportation barriers to health care access. J Commun Health. 2013;38(5):976-993. doi:10.1007/s10900-013-9681-1

19. Novara G, Checcucci E, Crestani A, et al. Telehealth in urology: a systematic review of the literature. how much can telemedicine be useful during and after the COVID-19 pandemic? Eur Urol. 2020;78(6):786-811. doi:10.1016/j.eururo.2020.06.025

20. Grad FP. Constitution of the world health organization. Bull World Health Organ. 2002;80(12):983-984.

21. Metzler I, Bayne D, Chang H, Jalloh M, Sharlip I. Challenges facing the urologist in low - and middle - income countries. World J Urol. $2020 ; 38$ (11):2987-2994. doi:10.1007/s00345-020-03101-6

22. Hoyler M, Finlayson SRG, McClain CD, Meara JG, Hagander L. Shortage of doctors, shortage of data: a review of the global surgery, obstetrics, and anesthesia workforce literature. World J Surg. 2014;38(2):269-280. doi:10.1007/s00268-013-2324-y

23. Chan SSW, Cheung NK, Graham CA, Rainer TH. Strategies and solutions to alleviate access block and overcrowding in emergency departments. Hong Kong Med J. 2015;21(4):345-352. doi:10.12809/hkmj144399

24. Gaughan J, Kasteridis P, Mason A, Street A. Why are there long waits at English emergency departments? Eur J Health Econ. 2020;21(2):209-218. doi:10.1007/s10198-019-01121-7

25. Al Kadhi O, Manley K, Natarajan M, et al. A renal colic fast track pathway to improve waiting times and outcomes for patients presenting to the emergency department. Open Access Emerg Med. 2017;9:53-55. doi:10.2147/OAEM.S138470

26. Gul M, Kaynar M, Yildiz M, et al. The increased risk of complicated ureteral stones in the era of COVID-19 pandemic. J Endourol. $2020 ; 34$ (8):882-886. doi:10.1089/end.2020.0658

27. Abdel Raheem A, Alowidah I, Soliman M, et al. Urolithiasis treatment options during COVID-19 pandemic: review of current recommendations and triage systems. Afr J Urol. 2020;26(1). doi:10.1186/s12301-020-00085-y

28. Türk C, Neisius A, Petrik A, Seitz C, Skolarikos A, Thomas K. EAU guidelines on urolithiasis. Eur Asso Urol. 2015;19:1-87.

29. Westphalen AC, Hsia RY, Maselli JH, Wang R, Gonzales R. Radiological imaging of patients with suspected urinary tract stones: national trends, diagnoses, and predictors. Acad Emerg Med. 2011;18(7):699-707. doi:10.1111/j.1553-2712.2011.01103.x

30. Kanzaria HK, Probst MA, Ponce NA. The association between advanced diagnostic imaging and emergency department length of stay. Am J Emerg Med. 2014;32(10):1253-1258. doi:10.1016/j.ajem201407038

31. Gottlieb RH, Erturk EN, Sotack JL, et al. CT in detecting urinary tract calculi: influence on patient imaging and clinical outcomes. Radiology. 2002;225:441-449. doi:10.1148/radiol.2252020101

32. Moore CL, Bomann S, Daniels B, et al. Derivation and validation of a clinical prediction rule for uncomplicated ureteral stone-the STONE score: retrospective and prospective observational cohort studies. BMJ. 2014;348:1-12. doi:10.1136/bmj.g2191

33. Smith-Bindman R, Aubin C, Bailitz J, et al. Ultrasonography versus computed tomography for suspected nephrolithiasis. $N$ Engl $J$ Med. $2014 ; 371$ (12):1100-1110.

34. Dubinsky TJT, Sadro CT. Acute onset flank pain-suspicion of stone disease. Ultrasound Q. 2012;28(3):239-240. doi:10.1097/ RUQ.0b013e318264f5e0

35. Doty E, DiGiacomo S, Gunn B, Westafer L, Schoenfeld E. What are the clinical effects of the different emergency department imaging options for suspected renal colic? A scoping review. J Am Col Emerg Physicians Open. 2021;2(3):e12446. doi:10.1002/emp2.12446

36. Gottlieb M, Hill ED, Arno K. Is point-of-care ultrasonography effective for the diagnosis of urolithiasis? Ann Emerg Med. 2019;73(5):517-519. doi:10.1016/j.annemergmed.2018.06.030

37. Argyropoulos A, Farmakis A, Doumas K, Lykourinas M. The presence of microscopic hematuria detected by urine dipstick test in the evaluation of patients with renal colic. Urol Res. 2004;32(4):294-297. doi:10.1007/s00240-004-0413-y

38. Papa L, Stiell IG, Wells GA, Ball I, Battram E, Mahoney JE. Predicting intervention in renal colic patients after emergency department evaluation. Can J Emerg Med. 2005;7(2):78-86. doi:10.1017/S1481803500013026

39. Steinberg PL, Nangia AK, Curtis K. A standardized pain management protocol improves timeliness of analgesia among emergency department patients with renal colic. Qual Manag Health Care. 2011;20(1):30-36. doi:10.1097/QMH.0b013e31820429d9

40. Holdgate A, Pollock T. Systematic review of the relative efficacy of non-steroidal anti-inflammatory drugs and opioids in the treatment of acute renal colic. Br Med J. 2004;328(7453):1401-1404. doi:10.1136/bmj.38119.581991.55

41. Kang C, Shu X, Herrell SD, Miller NL, Hsi RS. Opiate exposure and predictors of increased opiate use after ureteroscopy. J Endourol. 2019;33 (6):480-485. doi:10.1089/end.2018.0796

42. Motov S, Drapkin J, Butt M, Monfort R, Likourezos A, Marshall J. Pain management of renal colic in the emergency department with intravenous lidocaine. Am J Emerg Med. 2018;36(10):1862-1864. doi:10.1016/j.ajem.2018.07.021

43. Etchells E, O’Neill C, Bernstein M. Patient safety in surgery: error detection and prevention. World J Surg. 2003;27(8):936-941. doi:10.1007/ s00268-003-7097-2

44. Wong J, Khu KJ, Kaderali Z, Bernstein M. Delays in the operating room: signs of an imperfect system. Can J Surg. 2010;53(3):189-195.

45. Blackwell RH, Barton GJ, Kothari AN, et al. Early intervention during acute stone admissions: revealing "the weekend effect" in urological practice. J Urol. 2016;196(1):124-130. doi:10.1016/j.juro.2016.01.056

46. Jackson J, Farhi J, Wheeler K, Rueb G, Thom C, Schenkman N. Retrospective review of acute renal colic management in the emergency department and review of guidelines. Urol Pract. 2018;5(2):113-119. doi:10.1016/j.urpr.2017.02.007

47. Steinberg RL, Johnson BA, Antonelli J, Pearle MS. Urolithiasis in the COVID era: an opportunity to reassess management strategies. Eur Urol. 2020;78(6):777-778. doi:10.1016/j.eururo.2020.07.019 


\section{Publish your work in this journal}

Research and Reports in Urology is an international, peer-reviewed, open access journal publishing original research, reports, editorials, reviews and commentaries on all aspects of adult and pediatric urology in the clinic and laboratory including the following topics: Pathology, pathophysiology of urological disease; Investigation and treatment of urological disease; Pharmacology of drugs used for the treatment of urological disease. The manuscript management system is completely online and includes a very quick and fair peer-review system, which is all easy to use. Visit http://www.dovepress.com/testimonials.php to read real quotes from published authors.

Submit your manuscript here: https://www.dovepress.com/research-and-reports-in-urology-journal 